\title{
Telemedicine system for the care of patients with neuromuscular disease and chronic respiratory failure
}

Carlos Zamarrón ${ }^{1}$, Emilio Morete ${ }^{1}$, Francisco González ${ }^{2,3}$

${ }^{1}$ Division of Respiratory Medicine, Hospital Clínico Universitario, Santiago de Compostela, Spain

2Department of Physiology, Universidad de Santiago de Compostela, Santiago de Compostela, Spain

${ }^{3}$ Department of Surgery, Hospital Clínico Universitario, Santiago de Compostela, Spain

Submitted: 11 March 2013

Accepted: 30 May 2013

Arch Med Sci 2014; 10, 5: 1047-1051

DOI: 10.5114/aoms.2014.46223

Copyright @ 2014 Termedia \& Banach

\section{Abstract}

Introduction: Neuromuscular diseases cause a number of limitations which may be improved by using a telemedicine system. These include functional impairment and dependence associated with muscle weakness, the insidious development of respiratory failure and episodes of exacerbation.

Material and methods: The present study involved three patients with severe neuromuscular disease, chronic respiratory failure and long-term mechanical ventilation, who were followed up using a telemedicine platform. The telemedicine system is based on videoconferencing and telemonitoring of cardiorespiratory variables (oxygen saturation, heart rate, blood pressure and electrocardiogram). Two different protocols were followed depending on whether the patient condition was stable or unstable.

Results: Over a period of 5 years, we analyzed a series of variables including use of the system, patient satisfaction and clinical impact. Overall we performed 290 videoconference sessions, 269 short monitoring oximetry measurements and 110 blood pressure measurements. With respect to the clinical impact, after enrolment in the telemedicine program, the total number of hospital admissions fell from 18 to 3 .

Conclusions: Our findings indicate that the system was user friendly for patients and care givers. Patient satisfaction scores were acceptable. The telemedicine system was effective for the home treatment of three patients with severe neuromuscular diseases and reduced the need for hospital admissions.

Key words: home care, neuromuscular diseases, telemedicine.

\section{Introduction}

Telemedicine has been used in a variety of health care fields, but only recently with the advent of sophisticated technology has an interest among health professionals become evident. Spiraling health-care costs are forcing health-care providers to look for new cost-effective solutions for providing the best possible care. Information technology and, particularly, new ways of delivering care remotely through telecare interventions seem promising [1, 2].

Patients with long-term conditions represent a major health-care problem for public health-care systems. Neuromuscular diseases are chronic

\author{
Corresponding author: \\ Carlos Zamarrón MD \\ Servicio de Neumología \\ Hospital Clínico Universitario \\ de Santiago de Compostela \\ C/ Choupana s/n \\ 15706 Santiago de \\ Compostela, Spain \\ E-mail: carlos.zamarron. \\ sanz@sergas.es
}


conditions that may present progressive muscle wasting leading to loss of ambulation, difficulty swallowing, and respiratory muscle weakness.

Chronic respiratory failure in neuromuscular patients develops insidiously, and patient involvement is variable. Dyspnea is rare, due to the significant limitation of physical activity. Moreover, clinical signs of increased use of accessory muscles appear in advanced stages of the disease. Ventilatory failure develops gradually, with hypercapnia during sleep at first and then during daylight hours $[3,4]$.

Neuromuscular diseases associated with respiratory failure have specific characteristics. Their common feature is weakness of respiratory muscles, progression towards chronic respiratory failure and the eventual appearance of episodes of acute respiratory failure [5].

The peculiarities presented by these patients make it advisable to design and evaluate telemedicine systems that meet their needs. However, little information exists on the usefulness of this technology in patients with neuromuscular disease and chronic respiratory insufficiency.

The aim of this study is to describe the utility of a telemedicine system based on videoconferencing and telemonitoring of cardiorespiratory variables (oxygen saturation, heart rate, blood pressure and electrocardiogram) for the telecare of 3 patients with neuromuscular disease and chronic respiratory failure.

\section{Material and methods}

\section{Patients}

We monitored 3 patients over a period of 5 years with severe neuromuscular disease who were receiving long-term mechanical ventilation due to chronic respiratory failure. Two patients were having invasive mechanical ventilation by tracheotomy and the third patient was having non-invasive ventilation.

The first case involved a 68-year-old man diagnosed with scapular humeral dystrophy at the age of 30 . Starting in 1996, the patient was only able to walk a few steps with a cane and spent most of the day in a tailored sofa. Starting in 1990, he used a negative-pressure ventilator, a poncho wrap, and starting in 1999 he needed a positive-pressure ventilator by tracheostomy. Since 2005, he has been monitored at home with our telemedicine system.

The second case involves a 66-year-old woman with amyotrophic lateral sclerosis diagnosed in 2001. From 2003 to 2005 she was under non-invasive ventilation with satisfactory adaptation and clinical response. In April 2005 the patient presented several episodes of bronchoaspiration and respiratory distress, and afterwards she was unable to remain upright and walk, go out on her own, or use her hands and arms. In addition, she had difficulty swallowing and chewing. At the request of the patient, a percutaneous endoscopic gastrostomy and tracheostomy were performed. From 2005 to her death at the end of 2010, she needed constant mechanical ventilation via tracheostomy and was monitored at home with our telemedicine system.

The third case involves a 39-year-old man with Duchene disease. The patient needed a wheelchair and continuous support to move and perform basic tasks. Because of his nocturnal hypoventilation, he required non-invasive ventilatory support.

In this study, all patients gave informed written consent after protocol approval by the Local Ethics Research Committee.

\section{Telemedicine System architecture}

We used a commercially available Telemedicine System (Seguitel, Telefónica S.A., Madrid, 2005), which was modified to the monitoring needs of these patients. Within the system architecture, the Control Center has an Application Server and a Database Manager. The Control Center houses the service and governs the process by handling the database, user privileges, communications and security. The system allows a Telecare Service Center to perform remote monitoring of biological signals, videoconferencing, and to handle emergencies that may arise. Database, user privileges, communications and security were handled from the hospital by an administrator.

At the patient's home, there was a residential gateway, a set top box connected to the television as a user interface. Oximetry, blood pressure and electrocardiography were recorded for all patients, who were also equipped with a wireless alarm button to generate a system alarm call if needed. The patient could interact with the system at any time by accessing a simple menu on the TV screen. Most interactions involved input of biomedical data requested by the doctor or tutor nurse. System functionality is provided through the Web. Figure 1 shows how the system was configured at the patient's home.

Communications between client and server were protected by a system firewall against unauthorized access. In addition, the system also had a mechanism for user authentication and a password to check the role of the person connecting.

The tutor nurse visited the patient at home once a month, performed all regular procedures, and checked the equipment by testing all medical (sphygmomanometer, electrocardiograph, pulse oximeter and mechanical ventilator) and communication devices. Any malfunction was reported to the supervising physician. 


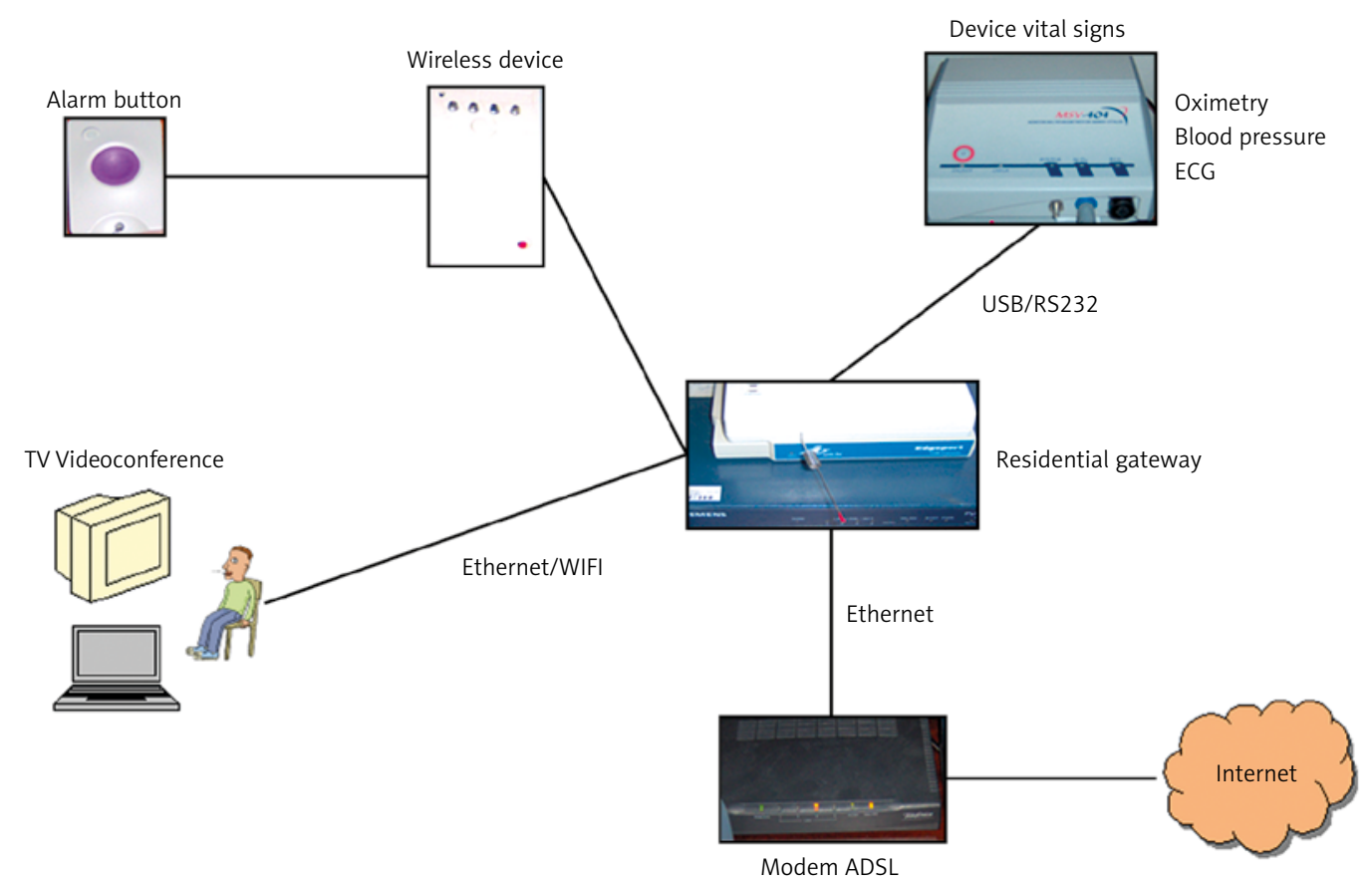

Figure 1. Telemonitoring system configuration in the patient's home

\section{Protocol}

Depending on the severity of the patient's condition, two different telecare protocols were followed. If the patient's condition was stable (baseline), a videoconference consultation, a 5-minute oximetry monitoring sample (Figure 2), and three blood pressure measurements were carried out every 2 weeks. If required, an electrocardiogram was also done. On the other hand, if the patient's condition became acute, similar procedures were carried out daily until stabilization. As these patients were on ventilators during sleep, an overnight oximetry control was carried out whenever necessary.

If the patient's condition worsened, the oxygen saturation was monitored overnight and reported the next morning to the physician. If the measurements were abnormal, the physician was informed and instructions were given to the patient's family to change the ventilator settings if needed.

These procedures were followed every time the patient requested a consultation.

\section{Variables}

- Use of system.

- Patient satisfaction by the Client Satisfaction Questionnaire (CSQ-8) [6]. This questionnaire includes eight items with four possible responses. Higher scores indicate greater satisfaction.

- Clinical impact was measured by the number of hospital admissions and consultations during the 5 years before inclusion in the telemedicine program as compared to after inclusion.
- Variation of basal saturation, oxygen desaturations, heart rate frequency, blood pressure and ECG. Regular monitoring of these parameters allowed us to obtain normal mean values for each patient as well as variation from baseline.

\section{Results}

\section{Use of the system}

The system was used on a continual basis, and blood pressure or oxygen saturation was measured at will. These measurements appeared on the TV screen and were viewed in the Control Center when patients felt symptoms worsening. The hospital alarm was activated through the system 39 times. During the 5-year monitoring period, we performed 290 videoconference sessions, 269 oximetry measurements and 110 blood pressure measurements.

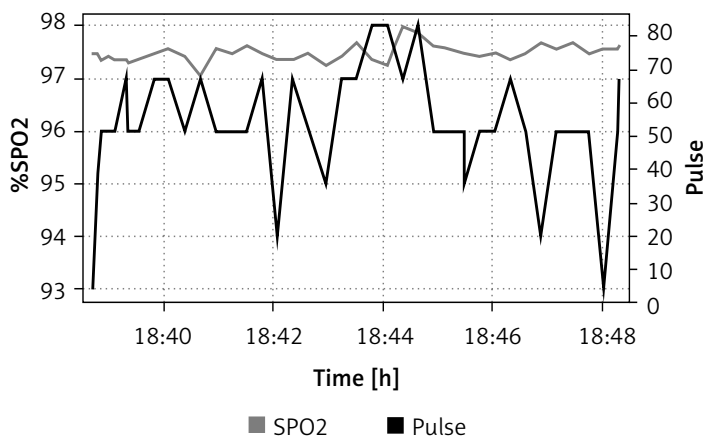

Figure 2. Data recorded using our home telemonitoring system. The graph shows oxygen saturation (black line) and heart rate (gray line) recorded during 8 min (from 18:40 to 18:48) 
The clinical information provided by patients, together with data from oximetry readings, made it possible to identify 15 exacerbations associated with infectious disease, but none required hospital admission. In addition, clinical data together with abnormal heart rhythm readings allowed us to diagnose heart problems in 2 cases.

The system is user friendly for patients and care givers. The equipment in the patients' residence is controlled with a device as simple as a TV remote control. Care givers only require about $4 \mathrm{~h}$ of training to learn how to use the system.

One of the most important problems was the lag between image and audio in videoconference sessions, which made it difficult to understand patients whose speech was already hindered by ventilators. No attempts by unauthorized users to gain access were detected.

\section{Patient satisfaction}

Using the Client Satisfaction Questionnaire (CSQ-8), patient 1 obtained a score of 21 , patient 2 a score of 18 , and patient 3 a score of 21 . The medium score was 20 . These scores indicate an acceptable level of satisfaction.

\section{Clinical impact}

The total number of hospital admissions by the 3 patients before enrolment in the telecare program was 18 , while after enrolment it dropped to 3 (patient 1 went from 3 admissions to 0 , patient 2 went from 5 to 0 , and patient 3 went from 10 to 3). The total number of hospital consultations before enrolment in the telecare program was 40, while after enrolment it dropped to 9 (patient 1 went from 15 consultations to 2, patient 2 went from 6 to 0 , and patient 3 went from 19 consultations to 7 ).

Although the system provides the possibility of interacting with primary care physicians, this feature was only used on limited occasions.

\section{Discussion}

The main finding of the present study is that our telecare system was effective for the home treatment of 3 patients with severe neuromuscular diseases, while reducing the need for home visits.

The functional limitation of patients with severe neuromuscular diseases and dependence associated with muscle weakness makes avoiding having to travel a crucial factor [7].

Respiratory monitoring is required to identify the real condition of these patients because their low mobility results in low clinical expression of breathlessness. In addition, these patients can experience the insidious development of respiratory failure. Oximetry monitoring with our telecare system detected changes in oxygen saturation and facilitated the detection of clinical deterioration and exacerbation. Thus, we found that exacerbation could be prevented by monitoring respiratory function. One of the biggest challenges currently facing public health systems is the care for patients with chronic respiratory diseases, and the interaction of training and intensity of work with patients with chronic diseases seems to have the most powerful effect on undertaking clinical and organizational self-management support practices. To facilitate clinicians' practice of self-management support for patients with chronic diseases it is very important to provide specialized patient care teams with professionals having complementary skills [8]. Some studies show that participation in an interactive workshop in preventive medicine increased the knowledge and the delivery rates of preventive procedures in the observed primary care practices [9]. Therefore, information technology and communication can play a crucial role facilitating coordination between different professionals. Telemedicine has the potential to become routine, presenting a new burden for health workers, who must adapt to new circumstances. The introduction of a home telecare system always requires organizational changes [10].

Early meta-analysis studies found that establishing systems for patient care was feasible using telecommunications technologies, but there was little evidence of clinical benefit as compared to face-to-face consultation [11]. However, subsequent studies have found telemedicine to be useful for monitoring chronic patients. Telecare in COPD appears to have a possible impact on the quality of life of patients and the number of times patients attend the emergency department and the hospital [12]. In our study, telemedicine was combined with face-to-face consultation once a month, which allowed us to see the patient in person and check the telemedicine equipment.

In patients with various chronic diseases, Hartvigsen Botsis reported patient satisfaction with home telecare but a preference for a combination with conventional methods [13]. In fact, our patients were satisfied with the system as shown by their Customer Satisfaction Questionnaire (CSQ-8) scores. Home telemonitoring of chronic diseases is a promising patient management approach that provides accurate and reliable data, empowers patients, influences their attitudes and behaviors, and potentially improves their medical conditions [14].

Patients with neuromuscular disease are often young subjects and, therefore, can more easily adapt to the daily use of technology. Providing effective homecare improves the quality of life, 
health and security of many chronically ill people and helps family members and friends care for them [15].

Horton evaluated a telecare system in 6 patients with COPD, and found that rapid access to care, an increased sense of personal safety, and continuity of care were seen as benefits. However, the equipment was perceived as complicated and bulky [16].

In contrast, our home equipment was perceived as user-friendly by both patients and healthcare personnel. The system can be accessed through a web interface from any computer or digital device equipped with a web browser. By involving a variety of doctors, such as primary care physicians, neurologists and pulmonary physicians, the system facilitates interaction between different departments and levels of health care, thereby reducing the workload of primary care. If necessary, pulmonary physicians could provide medical assistance to the primary physician. Integration of home telecare data with community-care information is essential for effective treatment [17].

We must keep certain study limitations in mind. Firstly, aspects of telemedicine systems evolved during the study period as a result of the rapid evolution of technology. Secondly, no telemedicine system has yet been recognized as the standard. Home care is currently one of the most empowering tools available to health administrators. Telemedicine prevents hospital admissions and inconvenience to neuromuscular patients. These systems also reduce dependence by increasing autonomy and self-monitoring of certain symptoms. It also facilitates integrated management, which may improve outcomes.

In our study, the combination of videoconference sessions and monitoring oximetric data allowed early detection of respiratory exacerbation and prevented the need for hospital admissions, because patients were treated without having to leave their home.

In conclusion, our findings indicate that the telecare system employed in the present study was user friendly for patients and care givers. Patients indicated a reasonable level of satisfaction. The telemedicine system was effective for the home treatment of three patients with severe neuromuscular diseases and reduced the need for hospital admissions.

\section{References}

1. McLean S, Sheikh A. Does telehealthcare offer a patient-centred way forward for the community-based management of long-term respiratory disease? Prim Care Respir J 2009; 18: 125-6.

2. Kuo YH, Chien YK, Wang WR, Chen $\mathrm{CH}$, Chen LS, Liu CK. Development of a home-based telehealthcare model for improving the effectiveness of the chronic care of stroke patients. Kaohsiung J Med Sci 2012; 28: 38-43.

3. MacDuff A, Grant IS. Critical care management of neuromuscular disease, including long-term ventilation. Curr Opin Crit Care 2003; 9: 106-12.

4. Sancho J, Servera E. Noninvasive ventilation for patients with neuromuscular disease and acute respiratory failure. Chest 2008; 133: 314-5.

5. Perrin C, Unterborn JN, Ambrosio CD, Hill NS. Pulmonary complications of chronic neuromuscular diseases and their management. Muscle Nerve 2004; 29: 5-27.

6. Larsen DL, Attkisson CC, Hargreaves WA, Nguyen TD. Assessment of client/patient satisfaction: development of a general scale. Eval Program Plann 1979; 2: 197-207.

7. Vitacca M, Comini L, Assoni G, et al. Tele-assistance in patients with amyotrophic lateral sclerosis: long term activity and costs. Disabil Rehabil Assist Technol 2012; 7: 494-500.

8. Kosmala-Anderson J, Wallace LM, Turner A. Does the professional and working context of United Kingdom clinicians predict if they use practices to support patients with long term conditions to self manage? Arch Med Sci 2010; 6: 815-21.

9. Gowin E, Avonts D, Horst-Sikorska W, Dytfeld J, Michalak M. Stimulating preventive procedures in primary care. Effect of PIUPOZ program on the deliveryof preventive procedures. Arch Med Sci 2012; 8: 704-10.

10. Ruggiero C, Sacile R, Giacomini M. Home telecare. J Telemed Telecare 1999; 5: 11-7.

11. Currell R, Urquhart C, Wainwright P, Lewis R. Telemedicine versus face to face patient care: effects on professional practice and health care outcomes. Cochrane Database Syst Rev 2000; 2: CD002098.

12. McLean S, Nurmatov U, Liu JL, Pagliari C, Car J, Sheikh A. Telehealthcare for chronic obstructive pulmonary disease. Cochrane Database Syst Rev 2011; 7: CD007718.

13. Botsis T, Hartvigsen G. Current status and future perspectives in telecare for elderly people suffering from chronic diseases. J Telemed Telecare 2008; 14: 195-203.

14. Pare G, Jaana M, Sicotte C. Systematic review of home telemonitoring for chronic diseases: the evidence base. J Am Med Inform Assoc 2007; 14: 269-77.

15. Robinson S, Stroetmann KA, Stroetmann VN. Tele-homecare for chronically ill persons: pilot trials, medical outcomes and future perspectives. Stud Health Technol Inform 2004; 103: 197-205.

16. Horton K. The use of telecare for people with chronic obstructive pulmonary disease: implications for management. J Nurs Manag 2008; 16: 173-80.

17. Rodriguez MJ, Arredondo MT, del Pozo F, Gomez EJ, Martinez A, Dopico A. A home telecare management system. J Telemed Telecare 1995; 1: 86-94. 